\title{
Surgical pulmonary embolectomy - an underestimated treatment option
}

\author{
Dariusz Zieliński, Andrzej Biederman \\ Cardiac Surgery Department, Medicover Hospital, Warsaw, Poland
}

Kardiochirurgia i Torakochirurgia Polska 2021; 18 (4): 260-261

It gave us great pleasure and interest to read the commentary of Zembala et al. on the article by Pruszczyk and Konstantinidis published in Kardiologia Polska [1, 2]. As outlined in the commentary, surgical embolectomy can provide excellent early and long-term outcomes.

According to ESC guidelines for patients with acute pulmonary embolism surgical embolectomy is recommended when other forms of treatment have failed or are not feasible. Patients with high or intermediate risk acute pulmonary embolism requiring urgent treatment are a very heterogeneous group. At present, the 3 existing alternative therapies - thrombolysis, catheter-based therapy, and surgical embolectomy - are difficult to compare. Perhaps a large registry will shed some more light on the results of these methods. However, several aspects regarding the treatment of patients with acute pulmonary embolism should be noted. DiChiacchio et al. reported that 35\% of patients undergoing surgical pulmonary embolectomy had evidence of chronic thromboembolic lesions at the time of surgery [3]. Regarding the challenging management of patients with chronic thromboembolic hypertension it is reasonable to assume that it may be important to treat these two similar but different diseases simultaneously, as it may have a direct impact on the outcome. According to our experience, which will be published shortly, about $30 \%$ of patients with acute pulmonary embolism had the presence of chronic thromboembolic lesions that are only surgically removable. These findings increase the risk of the operation, but it is probably still the best option.

Another issue is the lack of established and unambiguous management of patients with acute pulmonary embolism who present with "thrombi in transit" in the right heart cavities. Barrios found that the presence of thrombi in heart cavities results in a nearly 5 -fold increase in mortality [4]. Similarly, the RiHTER study showed that detection of "thrombi in transit" in the right heart cavities significantly increases mortality (19\% vs. $8 \% ; p=0.003)$ [5]. Similar results were reported by Burgos in a study evaluating the outcome of patients with thrombi in right heart cavities treated with thrombolysis or surgery (18.2\% vs. $18 \%$ ) [6]. However, the surgical method was not presented, and it is not known how complete the surgical embolectomy was, which is crucial for successful outcomes. In our experience of the surgical treatment of 20 consecutive patients with acute pulmonary embolism with "thrombi in transit" in the right heart cavities we achieved excellent results. All patients survived the operation, and at the 1-year follow-up there was no death. While previous guidelines mentioned the possibility of surgical treatment for these patients, the reasons for the disappearance in the current ESC guidelines are not known.

In our opinion, these two clinical conditions mentioned above strongly favor the surgical approach, but can only be compared to the results of the registries.

Finally, the important role of the pulmonary embolism response team (PERT) must be emphasized. These are teams that do not have a strictly defined membership. It usually involves intensive care, interventional and noninterventional cardiology experienced in the management of acute pulmonary embolism, radiology and other specialties as required. Implementation of PERT increases the use of advanced methods of treatment, particularly catheter directed therapies, but as a permanent member of this team, we have noted a significant increase in the number of surgical pulmonary embolectomies performed in recent years.

In conclusion, we share the opinion that the best treatment of unstable patients with acute pulmonary embolism, as well as selected intermediate high risk patients with an overloaded right ventricle, is surgical treatment, which in experienced surgical teams allows one to obtain excellent early and long-term results.

\section{Disclosure}

The authors report no conflict of interest.

\section{References}

1. Zembala M, Zawadzki F, Wajda-Pokrontka M, Filipiak K, Knapik P, Trejnowska E, Zembala M, Świerad M. Surgery over lysis in acute pulmonary embolism - let us challenge the guidelines. Kardiochir Torakochir Pol 2021; 18: 60-61.

2. Pruszczyk $P$, Konstantinides $S$. Where to treat patients with acute pulmonary embolism? Kardiol Pol 2020; 78: 15-19. 
3. DiChiacchio L, Pasrija C, Boulos FM, Ramani G, Jeudy J, Deatrick KB, Griffith BP, Kon ZN. Occult chronic thromboembolic disease in patients presenting for surgical pulmonary embolectomy. Ann Thorac Surg 2019; 108: 1183-1188.

4. Barrios D, Rosa-Salazar V, Morillo R, Nieto R, Fernández S, Zamorano JL, Monreal M, Torbicki A, Yusen RD, Jiménez D. Chest. Prognostic significance of right heart thrombi in patients with acute symptomatic pulmonary embolism: systematic review and meta-analysis. Chest 2017; 151: 409-416.
5. Koć M, Kostrubiec M, Elikowski W, Meneveau N, Lankeit M, Grifoni S, Kuch-Wocial A, Petris A, Zaborska B, Stefanović BS, Hugues T, Torbicki A, Konstantinides S, Pruszczyk P. Outcome of patients with right heart thrombi: the Right Heart Thrombi European Registry. RiHTER Investigators. Eur Respir J 2016; 47: 869-875.

6. Burgos LM, Costabel JP, Galizia Brito V, Sigal A, Maymo D, Iribarren A, Trivi M. Floating right heart thrombi: a pooled analysis of cases reported over the past 10 years. Am J Emerg Med 2018; 36: 911-915. 